\title{
UCRL-PROC-214921
}

LAWRENCE LIVERMORE N A T IO N A L LABORATORY

\section{Quantum-based Atomistic Simulation of Transition Metals}

J. A. Moriarty, L. X. Benedict, J. N. Glosli, R. Q. Hood, D. A. Orlikowski, M. V. Patel, P. Soderlind, F. H. Streitz, M. Tang, L. H. Yang

August 31, 2005

APS Conference on Shock Compression of Condensed Matter Baltimore, MD, United States July 31, 2005 through August 5, 2005 
This document was prepared as an account of work sponsored by an agency of the United States government. Neither the United States government nor Lawrence Livermore National Security, LLC, nor any of their employees makes any warranty, expressed or implied, or assumes any legal liability or responsibility for the accuracy, completeness, or usefulness of any information, apparatus, product, or process disclosed, or represents that its use would not infringe privately owned rights. Reference herein to any specific commercial product, process, or service by trade name, trademark, manufacturer, or otherwise does not necessarily constitute or imply its endorsement, recommendation, or favoring by the United States government or Lawrence Livermore National Security, LLC. The views and opinions of authors expressed herein do not necessarily state or reflect those of the United States government or Lawrence Livermore National Security, LLC, and shall not be used for advertising or product endorsement purposes. 


\title{
QUANTUM-BASED ATOMISTIC SIMULATION OF TRANSITION METALS
}

\author{
J. A. Moriarty, L. X. Benedict, J. N. Glosli, R. Q. Hood, D. A. Orlikowski, M. V. Patel, \\ P. Söderlind, F. H. Streitz, M. Tang and L. H. Yang
}

Lawrence Livermore National Laboratory, University of California, Livermore CA 94551

\begin{abstract}
First-principles generalized pseudopotential theory (GPT) provides a fundamental basis for transferable multi-ion interatomic potentials in $d$-electron transition metals within density-functional quantum mechanics. In mid-period bcc metals, where multi-ion angular forces are important to structural properties, simplified model GPT or MGPT potentials have been developed based on canonical $d$ bands to allow analytic forms and large-scale atomistic simulations. Robust, advancedgeneration MGPT potentials have now been obtained for Ta and Mo and successfully applied to a wide range of structural, thermodynamic, defect and mechanical properties at both ambient and extreme conditions of pressure and temperature. Recent algorithm improvements have also led to a more general matrix representation of MGPT beyond canonical bands allowing increased accuracy and extension to $f$-electron actinide metals, an order of magnitude increase in computational speed, and the current development of temperature-dependent potentials.
\end{abstract}

Keywords: Atomistic simulation, MGPT potentials, tantalum, molybdenum, equation of state, strength PACS: 71.15.Pd, 64.30.+t, 64.70.Dv, 62.20.-x

\section{INTRODUCTION}

Accurate and predictive large-scale atomistic simulation of materials properties is a forefront challenge in condensed-matter physics. There currently exists a wide spectrum of atomic-scale calculational methods, extending from exact quantum-mechanical techniques to classical descriptions with totally empirical force laws. All of these methods fall into one of two distinct categories separated by a material-dependent gap. On one side of this gap are electronic-structure methods based on direct quantum-mechanical treatments. These include quantum simulations that attempt to treat electron and ion motion on an equal footing, solving on the fly for both the electronic states of the system and the forces on the individual ions. In transition metals, methods based on modern density-functional theory (DFT)
[1] can provide a highly accurate description of the system and are chemically very robust, but they come at the price of being severely limited in the size $(\leq 1000$ atoms $)$ and duration $(\leq 10 \mathrm{ps})$ of the simulation. On the other side of the gap are methods used in atomistic simulations, such as molecular dynamics (MD), that treat only the ion motion, allowing much larger $\left(\sim 10^{8}-10^{10}\right.$ atoms $)$ and longer $(\sim 100 \mathrm{~ns})$ simulations by solving classical Newtonian equations of motion with the forces derived from explicit interatomic potentials. Bridging the electronic-atomistic gap is possible through the development of first-principles quantum-based potentials based on a systematic coarse-graining of the DFT electronic structure. In this paper, we present an overview of recent work at LLNL on developing quantum-based interatomic potentials for transition metals from densityfunctional theory and widely applying these 
potentials to the atomistic simulation of materials properties at both ambient and extreme conditions.

\section{QUANTUM-BASED POTENTIALS}

Within DFT quantum mechanics, firstprinciples generalized pseudopotential theory (GPT) provides a fundamental basis for $a b$ initio interatomic potentials in transition metals [2]. In the GPT a mixed basis of plane waves and localized $d$-state orbitals is used to self-consistently expand the electron density and total energy of the system in terms of weak $s p$ pseudopotential, $d-d$ tight-binding, and $s p-d$ hybridization matrix elements, which in turn are all directly calculable from first principles. For a bulk elemental metal, one obtains the real-space total-energy functional

$$
\begin{aligned}
& E_{\text {tot }}\left(R_{1} \ldots R_{N}\right)=N E_{v o l}(\Omega)+\frac{1}{2} \sum_{i, j}{ }^{\prime} v_{2}(i j ; \Omega) \\
& +\frac{1}{6} \sum_{i, j, k} v_{3}(i j k ; \Omega)+\frac{1}{24} \sum_{i, j, k, l} v_{4}(i j k l ; \Omega)
\end{aligned}
$$

The leading volume term in this expansion, $E_{v o l}$, as well as the two-, three-, and four-ion interatomic potentials, $v_{2}, v_{3}$ and $v_{4}$, are volume dependent, but structure independent quantities and thus transferable to arbitrary bulk ion configurations. The angular-force multi-ion potentials $v_{3}$ and $v_{4}$ reflect directional-bonding contributions from partially-filled $d$ bands and are generally important for the central transition metals. In the full $a b$ initio GPT, however, these potentials are multidimensional functions, so that $v_{3}$ and $v_{4}$ cannot be readily tabulated for application purposes. This has led to the development of a simplified model GPT or MGPT, which achieves short-ranged, analytic potential forms that can be applied to large-scale atomistic simulations $[3,4]$.

The MGPT is derived from the GPT through a series of systematic approximations applicable to mid-period transition metals. Canonical $d$ bands are introduced to express the $d$-state components of $v_{2}$ and the multi-ion potentials $v_{3}$ and $v_{4}$ analytically in terms of a single radial function and three universal angular functions that depend only on $d$ symmetry and apply to all transition metals and all volumes. To compensate for the approximations introduced into the MGPT, the $d$-state potential coefficients in $v_{2}, v_{3}$ and $v_{4}$ together with $E_{v o l}$ are constrained by fundamental theoretical and/or experimental data. In our current preferred scheme, we fit a combination of first-principles DFT calculations and experimental data on the cold equation of state, shear elastic moduli, unrelaxed vacancy formation energy and Debye temperature. Optimized Ta MGPT potentials, so obtained to $1000 \mathrm{GPa}[5,6]$, are displayed in Fig. 1 at equilibrium. Corresponding optimized Mo potentials, obtained to $400 \mathrm{GPa}$ [7], are also shown, as are earlier first-generation 1994 Mo potentials [4]. The new MGPT Mo potentials correct known problems with the latter at short bond lengths.
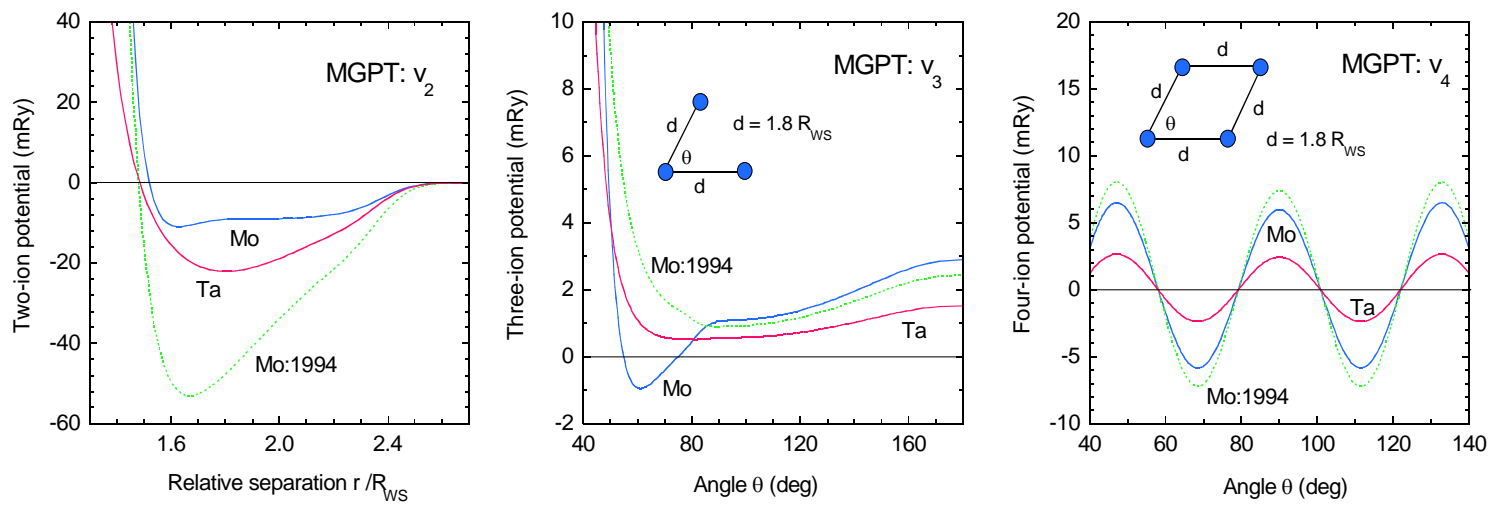

Figure 1. Optimized MGPT potentials $v_{2}, v_{3}$ and $v_{4}$ for Ta and Mo at their respective equilibrium volumes. Shown for comparison are earlier 1994 MGPT potentials for Mo. 


\section{SELECTED APPLICATIONS}

The MGPT potentials for Ta and Mo have been applied to a wide range of structural, thermodynamic, defect and mechanical properties at both ambient and extreme conditions. These properties include multiphase equation of state, melting and rapid resolidification, high-pressure elastic moduli and ideal strength, thermoelasticity and sound velocities, vacancy and self-interstitial formation and migration, grain-boundary structure, dislocation core structure and mobility, and the multiscale modeling of yield strength.

\section{Multiphase equation of state and melt}

We first discuss the quantum-based calculation of thermodynamics properties, with emphasis on the multiphase equation of state (EOS) and melting curve of Ta. In treating such properties, it is useful to break all thermodynamic quantities into cold (zero-temperature), ion-thermal and electronthermal contributions. Thus for the total pressure of at volume $\Omega$ and temperature $T$ we write:

$$
P(\Omega, T)=P_{0}(\Omega)+P_{\text {ion }}(\Omega, T)+P_{e l}(\Omega, T)
$$

with similar expressions for the internal energy $E(\Omega, T)$ and the Helmholtz free energy $A(\Omega, T)$. We treat the observed bcc solid, the liquid and the metastable A15 phase. The cold bcc components of our Ta EOS are taken from accurate firstprinciples DFT electronic-structure calculations obtained with the FP-LMTO method in the $0-1000$ GPa pressure range [8], and by construction, these components are exactly reproduced by the MGPT potentials. The ion-thermal EOS components are calculated from the MGPT potentials using quasiharmonic lattice dynamics in the low-T solid and MD simulation in the high-T solid and in the liquid. Good quality bec phonons are obtained and these yield a Grüneisen parameter with a nonlinear volume dependence at high pressure. The remaining electron-thermal EOS components are obtained by using MD/MGPT snapshots of finite-T structure in small-cell (27 or 54-atom) FP-LMTO calculations and then configuration averaging. In both the high-T solid and in the liquid only 10-15 configurations are needed at each volumetemperature point to obtain well-converged and smoothly varying results. At temperatures as low as melt, there are very significant electron-thermal contributions arising from the large density of electronic states at the Fermi level in mid-period transition metals such as Ta. Through direct MGPT simulations, we have also shown that the A15 structure becomes competitive with bcc at low pressures and high temperatures in $\mathrm{Ta}$, but over the entire $0-1000 \mathrm{GPa}$ range from $\mathrm{T}=0$ to melt, $\mathrm{bcc}$ is the predicted solid phase. A full two-phase bccliquid EOS and melt curve have been calculated over this pressure range and a temperature range of $0-35,000 \mathrm{~K}[5,9]$. The calculated $300-\mathrm{K}$ isotherm and Hugoniot from this EOS are shown in Fig.2 and compared against diamond-anvil-cell (DAC) and shock-compression experimental data $[10,11]$. Our corresponding equilibrium melt curve obtained from bcc and liquid free energies is displayed in Fig. 3 and compared against isobaric expansion data [12], the observed shock melting point [13] and recent laser-heated DAC measurements [14].

The pressure and temperature dependence of elastic moduli are closely related quantities with important implications for shock melting. As in Eq. (2), we calculate the single-crystal moduli $C_{i j}$ as a sum of cold, ion-thermal and electron-thermal

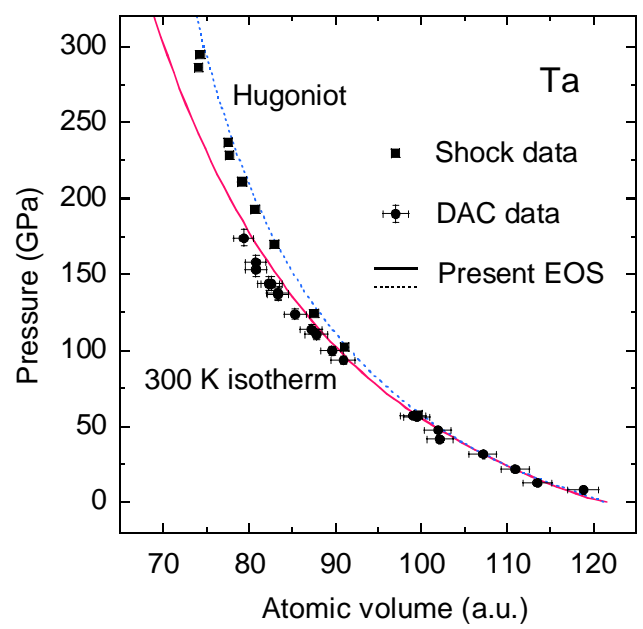

Figure 2. The $300-\mathrm{K}$ isotherm and Hugoniot for Ta, as calculated with the present multiphase EOS and as measured in DAC [10] and shock [11] experiments. 


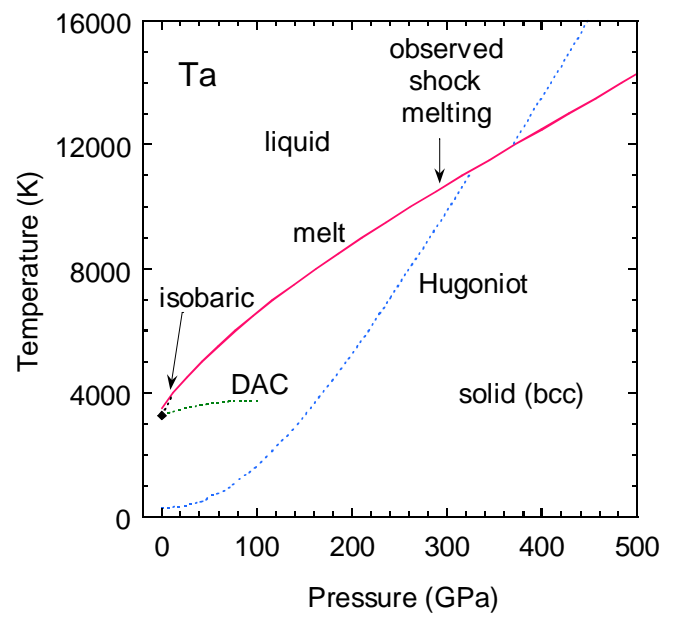

Figure 3. Present calculated melt curve (solid line) and predicted shock melting (intersection with dashed line) in Ta, as compared with experimental data [12-14].

components. The cold component is obtained from a blend of experimental data at low pressure and FP-LMTO calculations at high pressure and is reproduced by our MGPT potentials, while the electron-thermal component is calculated from FPLMTO calculations at finite temperature. The remaining the ion-thermal component is obtained from either of two MGPT-based methods. The first involves the calculation of explicit strain derivatives of quasiharmonic phonons, while the second involves exact Monte Carlo simulation and thereby also includes anharmonic contributions to $C_{i j}$. We have used these methods to investigate the full thermoelastic behavior of Ta and Mo at high temperature and pressure [15].

High-pressure melting in transition metals has become controversial with unexpectedly flat melt curves recently obtained in laser-heated DAC experiments for $\mathrm{Ta}$, Mo and other metals [14]. In contrast, we predict a steep melting curve for $\mathrm{Ta}$, which is consistent with both isobaric expansion data near ambient pressure [12] and the observed shock melting point near $300 \mathrm{GPa}$ [13]. Moreover, as shown in Fig. 4, our calculated sound velocities from high-T,P elastic moduli in bcc and liquid Ta are in good agreement with those measured along the Hugoniot in the shock melting experiments, confirming the interpretation of those experiments.

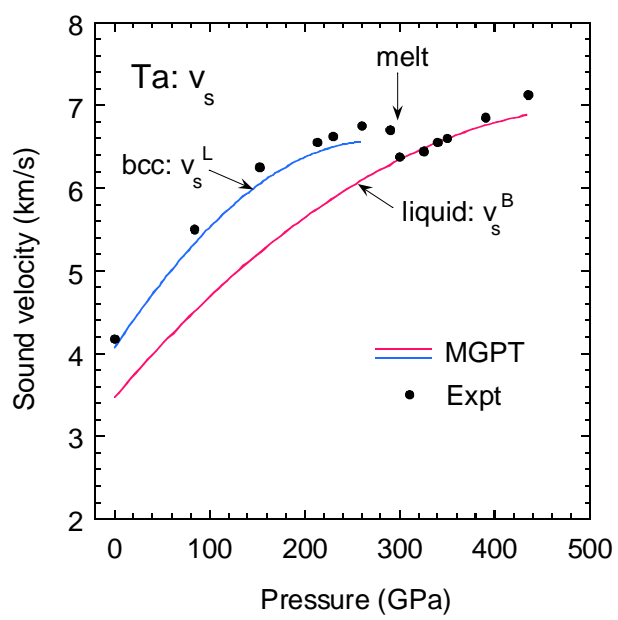

Figure 4. Calculated bcc and liquid Ta sound velocities from elastic moduli (solid lines) compared with values measured in shock melting experiments (points) [13].

\section{Multiscale modeling of yield stress}

The low-T and high-strain-rate plastic behavior of bcc transition metals is controlled by the intrinsic core properties of $a / 2\langle 111\rangle$ screw dislocations. Unlike the highly mobile edge dislocations in these metals, the motion of the screw dislocations is severely restricted by the nonplanar atomic structure of its core, resulting in low mobility, thermal activated kink pairs and a temperature-dependent yield stress.

For the accurate atomistic simulation of dislocation core properties, we have developed an advanced Green's function (GF) simulation method that allows both static and dynamic calculations [6]. Extensive GF/MGPT simulations have been carried out in Ta and Mo over wide ranges of pressure on the core structure, the Peierls stress and its stress-orientation dependence, and kink-pair energetics of $a / 2\langle 111\rangle$ screw dislocations $[6,16]$. In general, the core structure exhibits a three-fold directional spreading, of variable magnitude or polarization. We predict an isotropic core structure for Mo and $\mathrm{Ta}$ near equilibrium and into expansion, but an increasingly polarized core under hydrostatic pressure.

Similarly, our calculated Peirels stress $\tau_{P}$ in Ta and Mo exhibits a strong dependence on the 
orientation of the applied stress and large deviations from the well-known Schmid law. Our minimum $\tau_{P}$ in Mo at ambient pressure is close to the corresponding experimental estimate based on extrapolating the observed yield stress to zero temperature, and is a major improvement over that previously obtained with the 1994 MGPT potentials [17]. In the case of Ta, our minimum $\tau_{P}$ is still about a factor of two larger than the experimental estimate. In Ta and Mo we also find that both the ideal shear strength of the bcc perfect crystal and $\tau_{P}$ display approximate linear scaling with the $\langle 111\rangle$ shear modulus $G_{111}=\left(2 C^{\prime}+C_{44}\right) / 3$ to 1000 and $400 \mathrm{GPa}$, respectively. Our results for $\mathrm{Ta}$ are displayed in Fig. 5. These findings support the linear scaling of yield strength with shear modulus, as is assumed in most of the constitutive models used in the shock-compression community.

At finite temperature, the motion of the bcc screw dislocations occurs by the thermally assisted formation and migration of kink pairs. In microscale dislocation dynamics (DD) simulations of single-crystal plasticity for bcc metals [18], a key input quantity is the stress-dependent activation enthalpy for kink-pair formation, $\Delta H(\tau)$. This quantity controls the dislocation

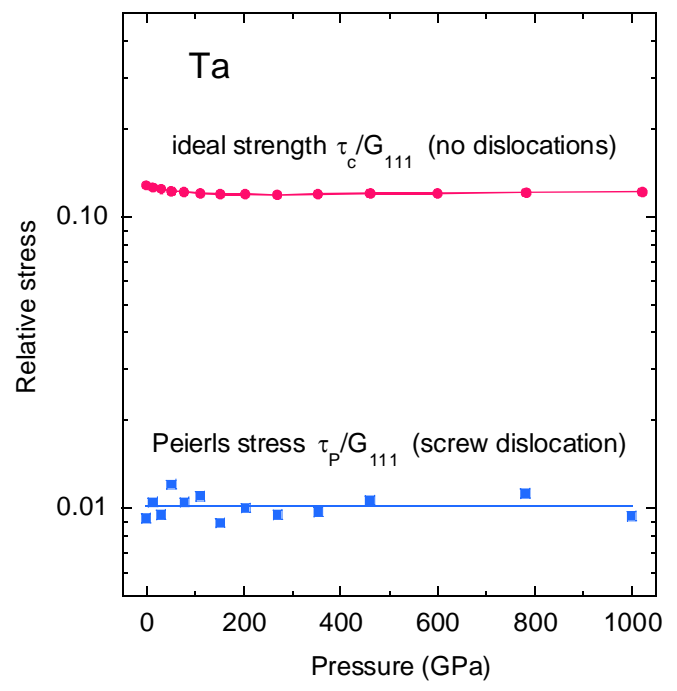

Figure 5. Calculated pressure scaling of the ideal shear strength and Peirels stress in Ta. mobility, with the stress-dependent velocity of a screw dislocation given by

$$
v_{\text {screw }}(\tau) \propto \exp \left[-\Delta H(\tau) / k_{B} T\right] .
$$

We have now performed extensive GF/MGPT atomistic simulations of $\Delta H(\tau)$ for both Ta and Mo over a wide range of pressures. These results can then be fitted to the simple analytic form used in the DD simulations. One can thereby import the required atomistic information directly into DD simulations for real materials at any assumed pressure condition. In Fig. 6 we display atomistically-informed DD simulations of yield stress in Ta as a function of temperature for three selected pressures. In the latter, we have everywhere scaled down the Peierls stress by about a factor of two to account for the overestimate noted above. Otherwise, the temperature dependence of the yield stress at ambient pressure is in good accord with experiment [19]. Similar DD simulations have also been done for Mo [16].

\section{BEYOND THE STANDARD THEORY}

One of the advantages of the first-principles GPT formalism for interatomic potentials is that it is systematically improvable in a manner consistent

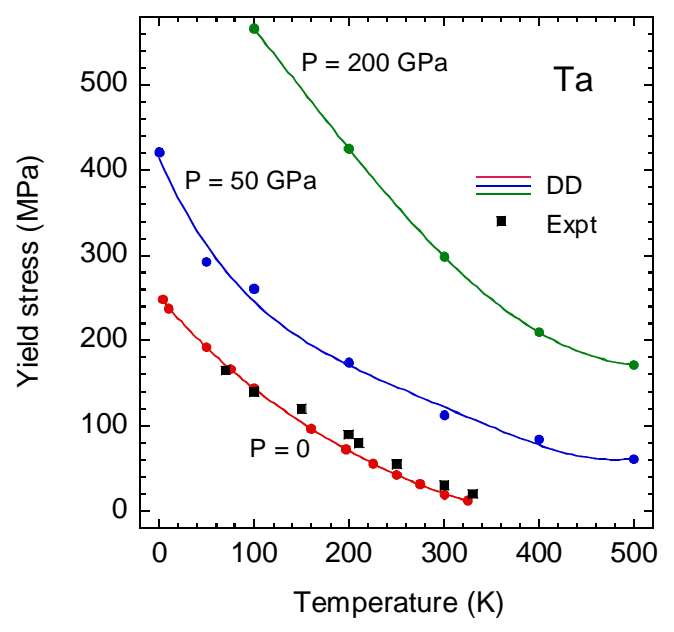

FIGURE 6. Atomistically-informed DD simulations of the single-crystal yield stress in bcc Ta at three pressures. 
with quantum mechanics. Recently, we have made major strides in this direction by developing a more general matrix MGPT representation. In the matrix MGPT, the three- and four-ion angular functions are recast as matrix products that can be evaluated on the fly numerically during a simulation. In this representation, several major extensions of the standard MGPT are possible. First, the theory can be immediately generalized to treat non-canonical $d$-bands with arbitrary tight-binding coefficients. Second, one may further generalize from $d$ states to $f$ states to treat actinide metals by replacing $5 \times 5 d$ state matrices with $7 \times 7 f$-state ones.

A third major advance that has been facilitated by the matrix MGPT is algorithm improvements in the evaluation of energies and forces, most notably an optimized strategy to calculate the multi-ion energy terms in Eq. (1) and an explicit analytic treatment of the forces [20]. This has resulted in MGPT simulation codes that are 6-10 times faster and has dramatically increased the size and scope of problems that can be addressed on large parallel platforms. The most striking example of this is the recent large-scale MD/MGPT simulations of rapid resoldification of Ta that have been performed on LLNL's new BlueGene/L supercomputer [21].

Two other important areas we are currently pursuing are the use of non-canonical bands and the development of temperature-dependent MGPT potentials. In principle, non-canonical bands permit a more accurate characterization of the underlying electronic structure. In practice, we have found them useful in improving the overall quality of the calculated phonon spectrum in certain difficult cases such as Mo. Interestingly, the introduction of just two non-canonical band parameters seems to be able to improve all phonons at all volumes. The concept of temperature-dependent potentials for $d$ - and $f$ electron metals is an important one because of the large electron-thermal effects at temperatures as low as melt. These effects are normally treated additively from the normal ion-thermal effects, as in Eq. (2), but we are now trying to capture them simultaneously and self-consistently by building MGPT potentials on the basis of the total electron free energy at finite temperature.

\section{ACKNOWLEDGEMENT}

This work was performed under the auspices of the U. S. Department of Energy by the University of California Lawrence Livermore National Laboratory under contract W-7405-ENG48.

\section{REFERENCES}

1. W. Kohn and L. J. Sham, Phys. Rev. 140, A1133 (1965).

2. J. A. Moriarty, Phys. Rev. B 38, 3199 (1988).

3. J. A. Moriarty, Phys. Rev. B 42, 1609 (1990).

4. J. A. Moriarty, Phys. Rev. B 49, 12431 (1994).

5. J. A. Moriarty, J. F. Belak, R. E. Rudd, P. Söderlind, F. H. Streitz and L. H. Yang, J. Phys.: Condens. Matter 14, 2825 (2002).

6. L. H. Yang, P. Söderlind and J. A. Moriarty, Philos. Mag. A 81, 1355 (2001).

7. J. A. Moriarty, unpublished.

8. P. Söderlind and J. A. Moriarty, Phys. Rev. B 57 10340 (1998).

9. J. A. Moriarty and P. Söderlind (to be published).

10. H. Cynn and C.-S. Yoo, Phys. Rev. B 59, 8526 (1999).

11. A. C. Mitchell and W. J. Nellis, J. Appl. Phys. 52, 3363 (1981).

12. J. W. Shaner, G. R. Gathers and C Minichino, High Temp.-High Pressures 9, 331 (1977).

13. J. M. Brown and J. W. Shaner, Shock Waves in Condensed Matter 1983, edited by J. R. Asay, R. A. Graham and G K. Straub (Elsevier, Amsterdam, 1984), p. 91.

14. D. Errandonea, B. Schwager, R. Ditz, C. Gessmann, R. Boehler and M. Ross, Phys. Rev. B 63, 132104 (2001); D. Errandonea, M. Somayazulu, D. Häusermann, and H. K. Mao, J. Phys.: Condens. Matter 15, 7635 (2003).

15. D. A. Orlikowski, P. Söderlind and J. A Moriarty (to be published).

16. L. H. Yang, P. Söderlind, M. Tang and J. A. Moriarty (to be published).

17. S. Rao and C. Woodward, Philos. Mag. A 81, 1317 (2001).

18. M. Tang, L. P. Kubin and G. R. Canova, Acta mater. 46, 3221 (1998).

19. W. Wasserbäch, Phil. Mag. A 53, 335 (1986).

20. J. N. Glosli, unpublished.

21. F. H. Streitz, J. N. Glosli and M. V. Patel (to be published). 